\title{
Carbonic anhydrase XII is a marker of good prognosis in invasive breast carcinoma
}

\author{
PH Watson*', SK Chia ${ }^{2}$, CC Wykoff ${ }^{3}$, C Han ${ }^{3}$, RD Leek ${ }^{3}$, WS Sly ${ }^{4}$, KC Gatter ${ }^{5}$, P Ratcliffe ${ }^{6}$ and AL Harris ${ }^{3}$ \\ 'Department of Pathology, D2 I 2-770 Bannatyne Avenue, University of Manitoba, Winnipeg, Manitoba, Canada R3E OW3; ${ }^{2}$ Division of Medical \\ Oncology, British Columbia Cancer Agency, Vancouver, British Columbia, Canada V5Z 4E6; ${ }^{3}$ Cancer Research UK, Molecular Oncology Laboratory, \\ University of Oxford, Institute of Molecular Medicine, John Radcliffe Hospital, Oxford OX3 9DU, UK; ${ }^{4}$ Edward A. Doisy Department of Biochemistry, \\ St Louis University School of Medicine, St Louis, MO 63104, USA; ${ }^{5}$ Nuffield Department of Clinical Laboratory Sciences, University of Oxford, John \\ Radcliffe Hospital, Oxford OX5 9DU, UK; ${ }^{6}$ Wellcome Trust Centre for Human Genetics, Oxford OX3 7BN, UK
}

\begin{abstract}
Hypoxia and $\mathrm{pH}$ influence gene expression in tumours, and it is becoming increasingly clear that the pattern of genes expressed by a tumour determines its growth and survival characteristics. Hypoxia-inducible factor-I (HIF-I) is a key mediator of the cellular response to hypoxia and high HIF-I expression has been identified as a poor prognostic factor in tumours. Recently, we identified the tumour-associated carbonic anhydrases (CA), CA9 and CA / 2 as hypoxia-inducible in tumour cell lines. Furthermore, we identified CA $\mathrm{IX}$ to be a poor prognostic factor in breast cancer. The aim of this study was to assess the prognostic significance of CA XII. CA XII expression was studied by immunohistochemistry in a series of 103 cases of invasive breast cancer and any association with recognised prognostic factors or relation with the outcome was examined. CA XII expression was present in 77 out of I03 (75\%) cases and was associated with lower grade $(P=0.00 \mathrm{I})$, positive estrogen receptor status $(P<0.00 \mathrm{I})$, and negative epidermal growth factor receptor status $(P<0.00 \mathrm{I})$. Furthermore, although CA XII expression was associated with an absence of necrosis $(P<0.00 \mathrm{I})$, expression of CA XII in some high-grade tumours was induced in regions directly adjacent to morphological necrosis. Additionally, using univariate analysis, CA XII positive tumours were associated with a lower relapse rate $(P=0.04)$ and a better overall survival $(P=0.0 \mathrm{I})$. In conclusion, CA XII expression is influenced both by factors related to differentiation and hypoxia in breast cancer in vivo and CA XII expression is associated with a better prognosis in an unselected series of invasive breast carcinoma patients. British Journal of Cancer (2003) 88, 1065-1070. doi:I0.1038/sj.bjc.6600796 www.bjcancer.com
\end{abstract}

(c) 2003 Cancer Research UK

Keywords: carbonic anhydrase; breast cancer; hypoxia; marker; prognosis

Methods for the detection of breast cancer have improved markedly over the past decade and patients are subsequently presenting with ever earlier stages of disease (Ernster and Barclay, 1997). Although established markers of tumour behaviour, including tumour size and nodal status are applicable for this group of patients, tissue-based markers that can predict the risk of progression and recurrence would improve the ability to identify those who would benefit from the treatment (Bennington et al, 1992; Ernster et al, 1996; Tabar et al, 2000). Indeed, several such markers, such as tumour grade and estrogen receptor (ER) status are well established. These factors mostly relate to cellular differentiation, while alternative indicators of the metabolic status of tumour cells, such as oxygenation, are relatively sparse (Brizel et al, 1997; Vaupel and Hoeckel, 1999). Necrosis is one such indicator of poor oxygenation and has been shown to be a prognostic indicator (Leek et al, 1999). However, necrosis is nonspecific, and may not always be associated with severe hypoxia (Mueller-Klieser et al, 1983; Parliament et al, 1997; Ramanujan et al, 2000). To identify endogenous hypoxic markers that might

*Correspondence: Dr P Watson; E-mail pwatson@cc.umanitoba.ca Received 23 August 2002; revised 25 November 2002; accepted 2 December 2002 have utility as prognostic indicators, we and others have begun to examine the expression patterns of known hypoxia-inducible genes in tumour specimens. Potential markers include the oxygenregulated subunits of the transcriptional complex hypoxiainducible factor-1 (HIF-1), a key mediator of the hypoxic response, HIF- $1 \alpha$ and HIF- $2 \alpha$, or HIF- 1 target genes (Shweiki et al, 1992; Maxwell et al, 1997; Zhong et al, 1999).

Recently, we extended the range of HIF-1 target genes by identifying the two tumour-associated transmembrane carbonic anhydrases (CA) CA9 (Opavsky et al, 1996; Ivanov et al, 1998) and CA12 (Ivanov et al, 1998; Tureci et al, 1998) as upregulated by hypoxia in a range of epithelial cancer cell lines (Wykoff et al, 2000). Carbonic anhydrases catalyse the reversible hydration of carbon dioxide to carbonic acid, providing a potential link between metabolism and $\mathrm{pH}$ regulation. We were therefore interested in examining the expression of these CA in tumourigenesis and progression in breast cancer. Our initial studies focused on CA IX as this gene demonstrated a more dramatic hypoxic induction in the tumour cell lines examined (Wykoff $e t a l$, 2000). CA IX expression was closely correlated with the presence of necrosis in both in situ and invasive cancer (Chia et al, 2001; Wykoff et al, 2001), and was found to be an independent predictor of relapse-free and overall survival (Chia et al, 2001). In the present study, we have investigated CA XII expression in breast cancer in 
the anticipation that its expression might also serve as an indicator of tissue hypoxia and tumour progression. Specifically, we wished to assess the pattern of expression in invasive breast carcinoma and the relation to outcome.

\section{MATERIALS AND METHODS}

\section{Patients and tissues}

A series of 103 surgically resected invasive breast carcinomas was assessed for CA XII expression in sections from paraffin tissue blocks. The clinicopathologic characteristics of this cohort are representative of that of a general population of breast cancer patients and are summarised in Table 1. The distribution of tumour types was $87 \%$ invasive ductal, $11 \%$ invasive lobular, and $2 \%$ special type (mucinous and tubular). All cases had previously been treated at the John Radcliffe Hospital and the Churchill Hospital, Oxford, and all cases underwent either modified radical mastectomy or lumpectomy with breast irradiation for primary treatment. Axillary lymph node positive cases also received adjuvant radiotherapy to the axillary region. Adjuvant systemic treatment consisted of endocrine therapy (for all postmenopausal women regardless of hormonal receptor status and comprising Tamoxifen at $20 \mathrm{mg}$ daily for 5 years) or chemotherapy (comprising six cycles of intravenous cyclophosphamide, methotrexate and 5-fluorouracil delivered every 3 weeks). All patients were assessed by follow-up every 3 months for the first 18 months and then every

Table I Clinical and pathological characteristics

\begin{tabular}{|c|c|}
\hline Patient characteristics & Number \\
\hline Total patients & 103 \\
\hline Age (median, range) (years) & $59(28-83)$ years \\
\hline$<50$ & 27 \\
\hline$>50$ & 76 \\
\hline \multicolumn{2}{|l|}{ Surgical treatment } \\
\hline Lumpectomy & 5 \\
\hline Lumpectomy+RT & 63 \\
\hline Mastectomy & 35 \\
\hline Tumor size (median, range) $(\mathrm{cm})$ & $2.4 \mathrm{~cm}(0.8-8 \mathrm{~cm})$ \\
\hline$<2$ & 43 \\
\hline$>2$ & 58 \\
\hline \multicolumn{2}{|l|}{ Node status } \\
\hline Positive (number positive nodes) & $58(1-16)$ \\
\hline Negative & 45 \\
\hline \multicolumn{2}{|l|}{ Grade } \\
\hline I & 15 \\
\hline 2 & 48 \\
\hline 3 & 40 \\
\hline \multicolumn{2}{|l|}{ ER status } \\
\hline Positive & 70 \\
\hline Negative & 33 \\
\hline \multicolumn{2}{|l|}{ EGFR status } \\
\hline Positive & 56 \\
\hline Negative & 46 \\
\hline \multicolumn{2}{|l|}{ Adjuvant therapy } \\
\hline Chemotherapy & 27 \\
\hline Hormonal therapy (Tamoxifen) & 80 \\
\hline Median duration follow-up (median, range) & $6.2(0.4-10.1)$ \\
\hline Relapses & 41 \\
\hline Deaths & 32 \\
\hline
\end{tabular}

6 months thereafter. Treatment for confirmed recurrent disease was by endocrine therapy for soft tissue or skeletal metastasis or by chemotherapy for visceral disease and failed endocrine therapy.

\section{Assessment of tumour grade, necrosis, and hormone receptor status}

Tumour grade and necrosis was scored on haematoxylin- and eosin-stained sections immediately adjacent to those sections subjected to immunohistochemistry. Tumour grade was assessed using the Nottingham system (Elston and Ellis, 1991). The entire section was also assessed for the percentage of necrosis present within the invasive tumour component. Both parameters were scored by a single pathologist (PHW) independently from the IHC analysis and blinded to the cohort's clinical data and outcome. Necrosis was assessed semiquantitatively by estimating the area of the tumour section involved by light microscopy. Necrosis within in situ carcinoma components was not scored. For statistical analysis the percentage of necrosis was either assessed as a continuous variable or divided into negative or positive (where the presence of any necrosis was considered positive). Estrogen receptor (ER) analysis was performed using an enzyme linked immunosorbent assay technique (Abbott Laboratories, Chicago, USA). Epidermal growth factor receptor (EGFR) was determined using ligand binding of $\left[{ }^{125} \mathrm{I}\right]$ EGF to tumour membranes (Fox et al, 1994). Tumours with an EGFR level greater than or equal to $20 \mathrm{fmol} \mathrm{mg}^{-1}$ of membrane protein and ER greater than or equal to $10 \mathrm{fmol} \mathrm{mg}^{-1}$ of cytosol protein were considered positive.

\section{Immunohistochemistry (IHC)}

Immunohistochemical staining for CA XII was performed on $5 \mu \mathrm{m}$ serial sections on coated slides from paraffin-embedded blocks. The anti-CA XII antibody (Kivela et al, 2000a; Tureci et al, 1998) has previously been characterised and we have also further confirmed its ability to specifically detect CA XII expression in tissue sections by correlation with Western blot analysis in human breast tumour specimens (Wykoff et al, 2001). All slides were first deparaffinised and then placed in $0.5 \%$ hydrogen peroxide for $15 \mathrm{~min}$ to saturate endogenous peroxidases. Incubation with $10 \%$ normal human serum in Tris buffered saline (TBS) for $15 \mathrm{~min}$ was then performed to block nonspecific uptake of the antibody. The anti-CA XII antibody (Kivela et al, 2000a) was used at a dilution of $1: 50$ in TBS with $5 \%$ normal human serum for 30 min. Next, a $30 \mathrm{~min}$ incubation with a peroxidase conjugated to goat antimouse immunoglobulins (Dako EnVision+System, Peroxidase, Mouse; Dako, Carpinteria, CA, USA) was performed. Slides were then stained with 3,3-diaminobenzidine chromogen solution for $8 \mathrm{~min}$ and then counterstained with haematoxylin and mounted with aquamount. All staining was performed on an automated immunohistochemical stainer (MiniPrep 75, Tecan; Reading, UK) at room temperature (RT). Following successive incubations (except the normal human serum block) the slides were washed twice with TBS for $5 \mathrm{~min}$.

\section{Assessment of CA XII expression}

Immunostaining for CA XII was quantified by light microscopy and semiquantitative scoring by one author (PHW) blinded to the cohort's clinical data and outcome. In brief, a score of $0-3$ was assigned for the intensity of staining assessed at low-power magnification ( $\times 10$ objective; 0 : no staining; 1 : weak staining; 2 : moderate staining; and 3: strong staining). The percentage of invasive tumour cells that were stained positive was then estimated at both low and high magnification and the product of the intensity of staining and the percentage of tumour positive cells was then calculated to produce an immunostaining score (IHC score) of $0-300$. 


\section{Statistical analysis}

For statistical analysis CA XII expression was evaluated as a continuous variable using the IHC-score. CA XII was also assessed as a categorical variable using the median (IHC score $>40$ ) to distinguish low from high expression. CA XII was evaluated in relation to a range of established prognostic variables using Mann - Whitney and Kruskal-Wallis tests where appropriate. The association with relapse-free and overall survival was assessed by univariate (log-rank test and Kaplan-Meier method) and multivariate (Cox's regression model) analysis.

\section{RESULTS}

\section{CA XII expression in relation to clinicopathological variables}

The specificity of the rabbit anti-human CA XII antisera for detection of CA XII in breast tissue sections was initially confirmed by comparison of IHC scores with the detection of $46-48 \mathrm{kDa}$ bands corresponding to CA XII by immunoblotting performed on the same tumour specimens (Wykoff et al, 2001). CA XII expression was observed in 77 out of 103 (75\%) cases of invasive carcinoma with a wide distribution of IHC scores (range 0-270, median 40, mean 65). Immunostaining was restricted to membranous staining of epithelial tumour cells and the pattern of expression was usually homogeneous. Representative examples of tumours showing overall low, moderate and high CA XII expression are illustrated in Figure 1.

CA XII expression was compared with several established clinical-pathological prognostic variables. When CA XII was assessed as a continuous variable, there was a strong correlation between CA XII expression and tumour grade $(r=-0.39$, $P<0.0001$ Spearman's test), ER level $(r=0.53, P<0.0001)$, EGFR $(r=-0.38, \quad P<0.0001)$ and extent of necrosis $(r=-0.44$, $P<0.0001)$. When CA XII was assessed as a categorical variable these relations were also evident, with high CA XII expression significantly associated with lower tumour grade, positive ER
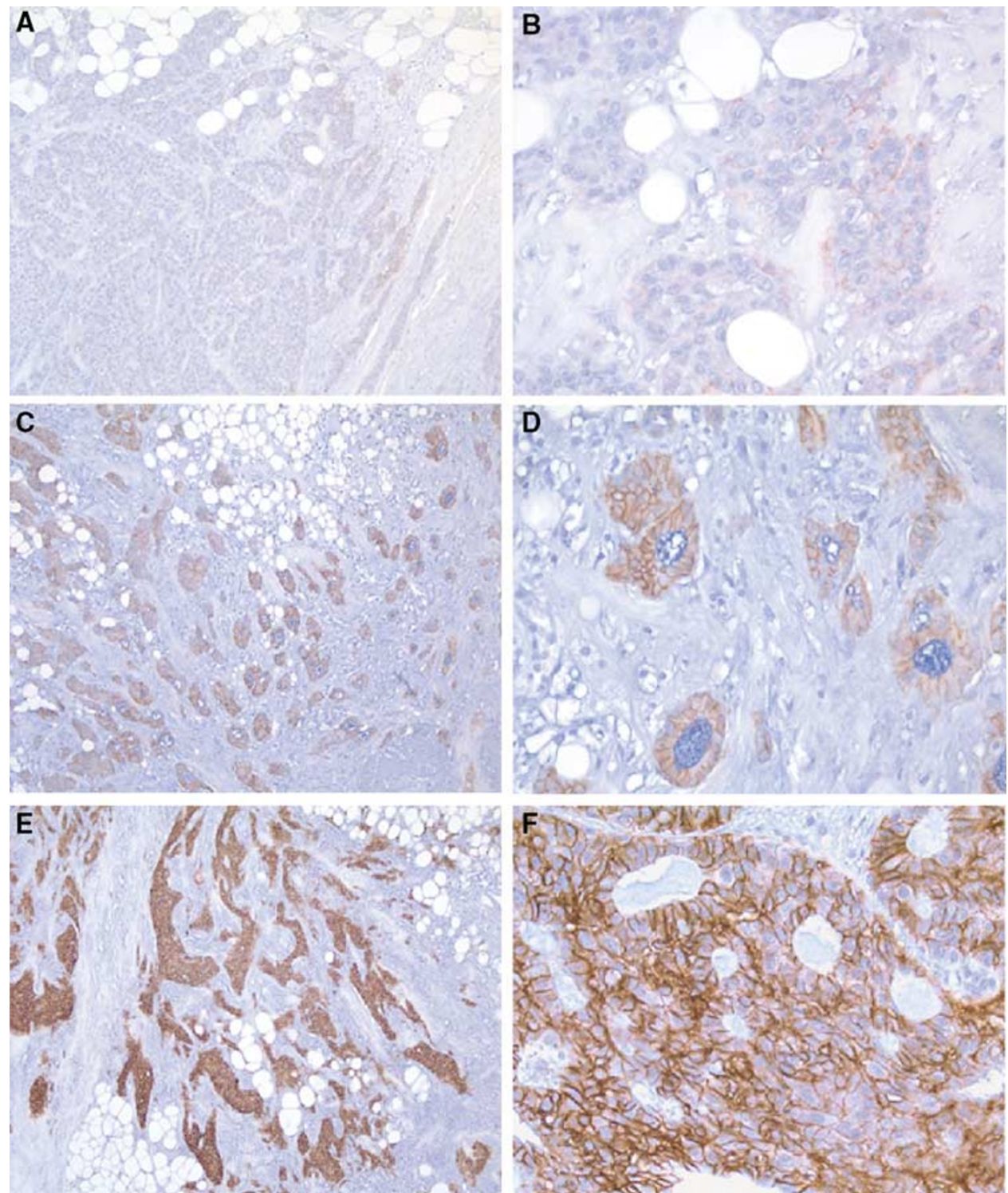

Figure I Expression of CA XII in invasive breast tumours. The panels show representative examples of CA XII expression detected by IHC in three invasive ductal carcinomas. The cases were associated with overall IHC scores of 10 (A and $\mathbf{B}), 100(\mathbf{C}$ and $\mathbf{D})$, and 225 (E and $\mathbf{F})$ respectively. Panels on the right side (B, D and $\mathbf{F}$ ) are higher magnifications to show cellular detail within the corresponding tumour shown on the left. Original magnifications; left $\times 40$, right $\times 200$. 

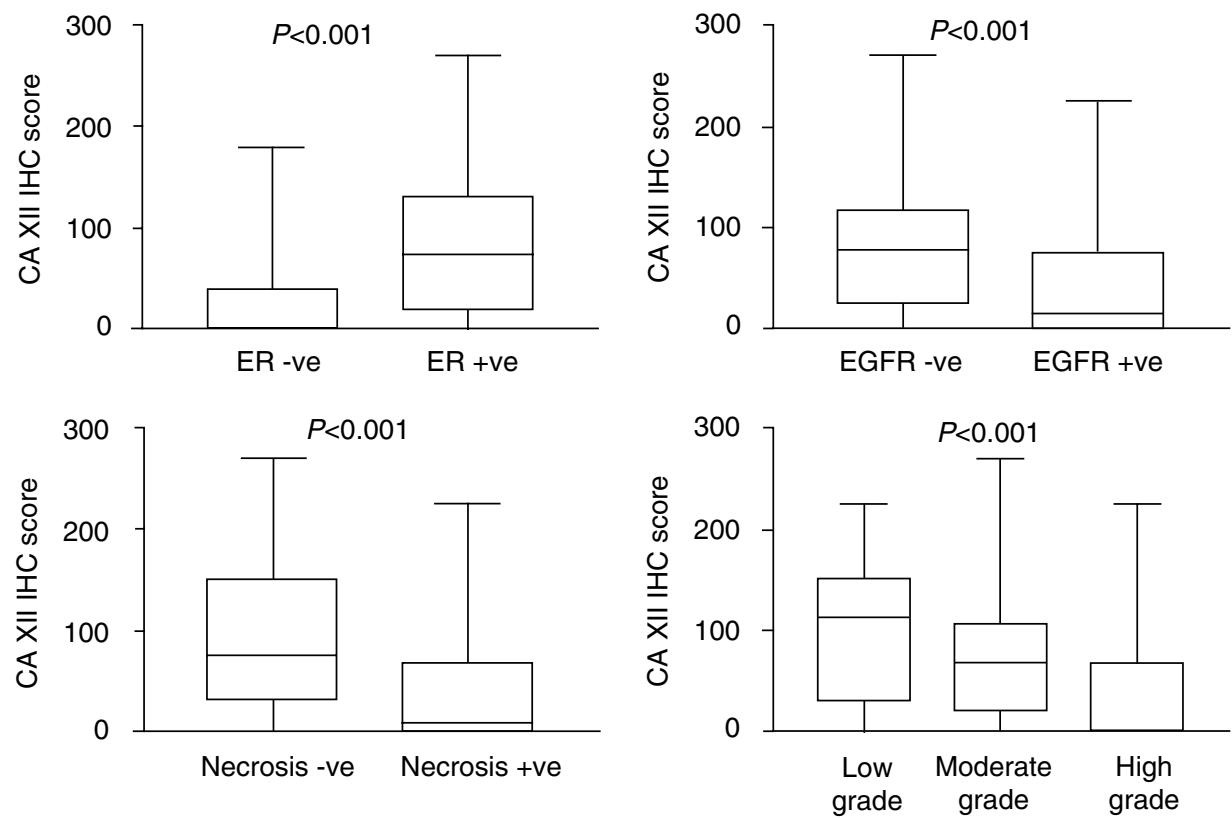

Figure 2 Association of CA XII with ER, EGFR, necrosis and grade. Each box indicates the range of CA XII IHC score from the 25th percentile to the 75th percentile, the line indicates the median, and the whiskers above and below the box show the highest and lowest values.

status, negative EGFR status, and the absence of necrosis (Figure 2). Despite the negative association between overall CA XII staining and necrosis, accentuation of CA XII expression was sometimes observed in tumour cells immediately adjacent to areas of necrosis within both in situ and invasive components (Figure 1), as might be predicted from our previous in vitro studies with tissue-culture cells (Wykoff et al, 2000). However, this expression pattern was typically present only focally in high-grade tumours where the overall level of CA XII within the tumour was low. No other significant relation with other prognostic variables such as age, nodal status, tumour size, or tumour type were observed.

\section{Relation of CA XII expression to relapse-free survival and overall survival}

Univariate analysis was conducted to explore the relation of CA XII to established prognostic factors and to survival. The relation of several conventional factors with survival in this cohort of cases was first confirmed. Nodal status, grade, and ER status were all significantly related to both relapse-free survival $(P$ values $=<0.001,0.04,0.03$, respectively $)$ and overall survival $(P$ values $=<0.001,0.04,0.02$, respectively) in this series. In addition, tumour size (relapse-free survival, $P=0.04$ ) and patient age (overall survival, $P=0.05$ ) were also predictive of aspects of survival. Further analysis, considering CA XII expression as a categorical value (IHC score 0, CA XII negative; IHC score $>0$, CA XII positive), showed a significant association between CA XII positive tumours and a longer relapse-free survival $(P=0.04)$ and a better overall survival $(P=0.01)$ (Figure 3$)$. In addition, subgroup analysis showed that the presence of CA XII expression was also a significant predictor of both longer relapse-free and better overall survival for tumours without necrosis $(P=0.04,0.04$, Figure 4).

We were unable to demonstrate an independent influence in multivariate Cox's proportional hazard analysis when CA XII, lymph node status, grade, ER status and necrosis were all
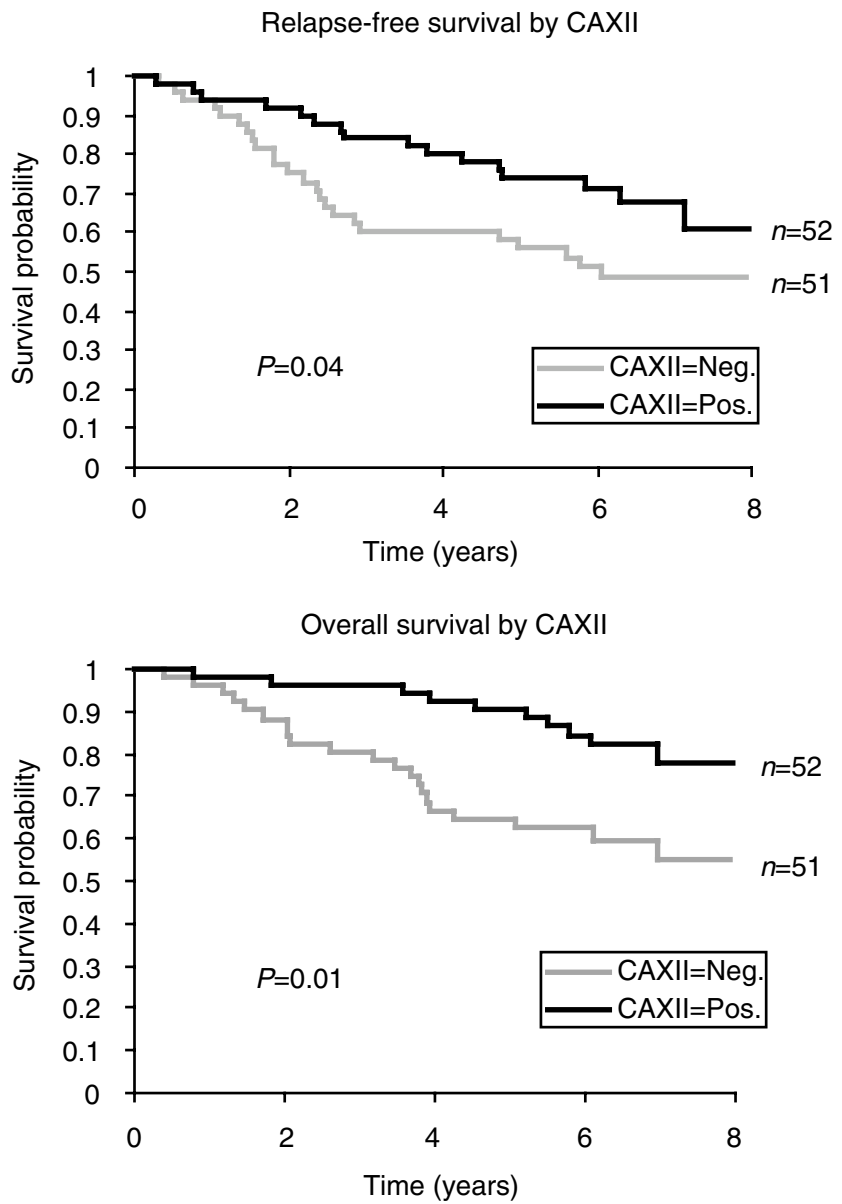

Figure 3 Kaplan - Meier curves demonstrating a relation between CA $\mathrm{XII}$ and relapse-free and overall survival within the entire cohort. 
Relapse-free survival by CAXII in the absence of necrosis

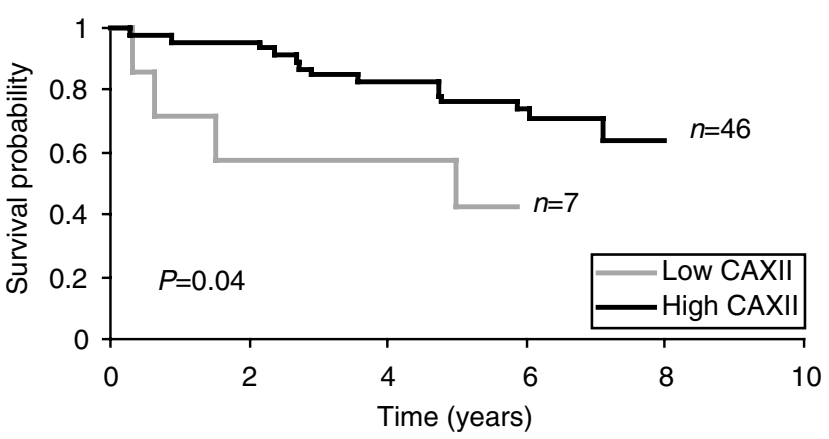

Overall survival by CAXII in the absence of necrosis

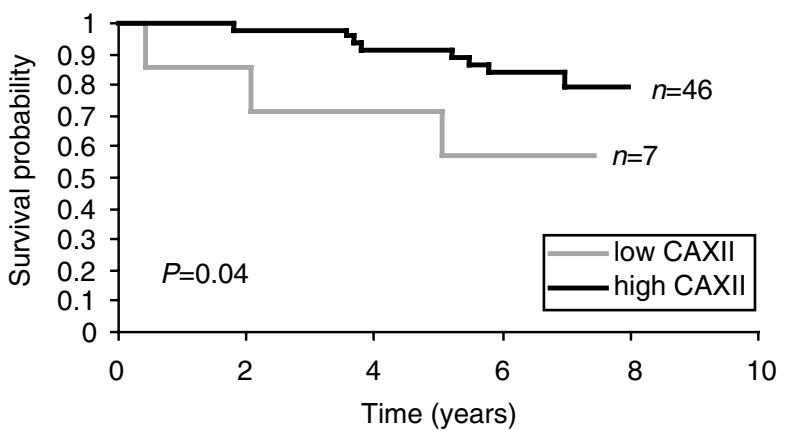

Figure 4 Kaplan - Meier curves demonstrating an association of CA XII with outcome within subgroups of tumours with and without necrosis.

considered in the model. However it should be noted that amongst these factors, only lymph node status emerged as an independent predictor of survival in this cohort of patients.

\section{DISCUSSION}

In this study, we have shown that CA XII is frequently expressed in invasive breast carcinoma, where expression is strongly associated with several good prognostic parameters, including low tumour grade, ER-positive status, EGFR-negative status, and absence of necrosis.

These findings extend our previous observations that expression of CA XII is closely related to tumour differentiation in preinvasive in situ ductal breast carcinomas (DCIS), where CA XII was observed to be highest in well differentiated and reduced in poorly differentiated lesions (Wykoff et al, 2001). Furthermore, although CA XII expression was generally low or absent in high-grade tumours with necrosis, we observed that focal induction of CA XII can be present in tumour cells adjacent to necrosis. This is consistent with our previous observations in in situ breast carcinoma (Wykoff et al, 2001) and similar observations made by others in invasive breast carcinoma (Ivanov et al, 2001), and with our in vitro findings that CA XII is regulated to some degree by hypoxia in breast tumour cells (Wykoff et al, 2000).

CA XII is widely expressed in specialised cells within normal tissues (Ivanov et al, 2001) and in several different tumour types (Tureci et al, 1998; Kivela et al, 2000a, b; Ivanov et al, 2001). In one previous study of 29 breast cancers, CAXII was found to be focally expressed in normal breast and expressed in $62 \%$ of invasive carcinomas, primarily in low-grade tumours (Ivanov et al, 2001).
In the normal colon and associated cancers, CA XII expression was frequently found in superficial regions of normal colonic mucosal epithelium, and was found to persist at comparable levels within preinvasive and invasive lesions (Kivela et al, 2000a). Although no significant association was seen with tumour grade, it should be noted that relatively small subgroups were available for analysis of invasive carcinoma and that the cranial-caudal location of each lesion may also have influenced expression. Despite this, a trend was observed towards reduced expression in high- $v$ s low-grade adenoma, complementing our findings in preinvasive breast cancer (Wykoff et al, 2001). In contrast to this pattern of decreasing expression with increasing grade, Kivela et al (2000a) observed increased expression in nonsurface, deep cryptal epithelium with progression from normal through in situ to invasive disease, between low- and high-grade adenomas, and in higher stage invasive tumours. It is interesting to speculate that both these regional differences and the increased expression of CA XII in deeper regions within adenomas and colon carcinomas might relate to differences in vascularity, oxygenation and relative hypoxia.

Although other members of the CA gene family have been examined in several tumour types (Nogradi, 1998), and it is known that the increased expression of CA IX is associated with aspects of early tumourigenesis in cervix, colon and lung tumours (Liao and Stanbridge, 1996; Saarnio et al, 1998; Vermylen et al, 1999), a prognostic role for CA has not previously been identified in breast cancer. Our recent studies have shown that increased CA IX expression is associated with poor relapse free and overall survival in invasive breast cancer (Chia et al, 2001). CA IX expression is also closely related to necrosis. Necrosis may be attributable to several factors (Mueller-Klieser et al, 1983; Parliament et al, 1997; Ramanujan et al, 2000), but an important factor is likely to be hypoxia and the relation between CA IX and necrosis is consistent with the recently described relation between HIF- $1 \alpha$ and prognosis in tumours (Zhong et al, 1999). However, the results of the current study indicate that while hypoxia may influence CA XII expression in focal areas discernable within high-grade breast tumours, the regulation of CA XII by differentiation-related factors appears to be dominant in vivo. Consequently, CA XII is associated with several phenotypic characteristics consistent with well differentiated tumours and good overall survival, at least in univariate analysis. The relatively small size of the series limited the capacity to study CA XII and prognosis within subsets of tumours with otherwise good prognosis. However, CA XII appears also to be a discriminator of good outcome within tumours that are negative for necrosis. Our results suggest that further examination of the role of CA XII as a prognostic marker may be warranted within certain subgroups of patients. The possibility that CA XII expression may also be relevant to chemotherapy response in such cases should also be considered, since an acidic extracellular $\mathrm{pH}$ can reduce uptake and efficacy of doxorubicin (Raghunand et al, 1999).

In conclusion, we have shown that CA XII is frequently expressed in invasive breast carcinoma and that focal enhancement of expression can be observed adjacent to areas of necrosis, supporting the notion that CA XII is a hypoxia regulated gene in vivo. However, the dominant factors in CA XII regulation are clearly related to tumour differentiation and higher levels of CA XII expression are associated well with differentiated tumours and with a better relapse-free and overall survival. In view of the known differences in extracellular pH (Gerweck, 1998) and the contrasting patterns of expression observed for CA IX and XII in normal and neoplastic breast tissues (Wykoff et al, 2001), it will be important to explore the prognostic significance of CA XII further, in concert with other carbonic anhydrases and in relation to specific chemotherapies that may be influenced by extracellular $\mathrm{pH}$ (Raghunand et al, 1999), in larger prospective studies. 


\section{ACKNOWLEDGEMENTS}

The authors would like to thank Mrs Lesley Richards for secretarial assistance with this manuscript. This work was supported by the Imperial Cancer Research Fund and the Wellcome Trust. PHW is supported by a Scientist Award from the Medical Research Council of Canada, an Academic Award from the US Army Medical Research and Materiel Command (USAMRMC), and a Research Travel Fellowship from Burroughs Welcome. SKC is supported by the Shane Fellowship and the Canadian Breast Cancer Foundation - British Columbia/Yukon Chapter.

\section{REFERENCES}

Bennington JL, Lagios MD, Margolin FR (1992) Impact of mammographic screening on the size and the relative frequency of invasion in breast cancers seen in a community hospital from 1975-1988. Pathology (Phila) 1: $11-21$

Brizel DM, Sibley GS, Prosnitz LR, Scher RL, Dewhirst MW (1997) Tumour hypoxia adversely affects the prognosis of carcinoma of the head and neck. Int J Radiat Oncol Biol Phys 38: 285-289

Chia SK, Wykoff CC, Watson PH, Han C, Leek RD, Pastorek J, Gatter KC, Ratcliffe P, Harris AL (2001) Prognostic significance of a novel hypoxia regulated marker - carbonic anhydrase IX in invasive breast carcinoma. $J$ Clin Oncol 19: $3660-3668$

Elston CW, Ellis IO (1991) Pathological prognostic factors in breast cancer. I. The value of histological grade in breast cancer: experience from a large study with long-term follow-up. Histopathology 19: 403-410

Ernster VL, Barclay J (1997) Increases in ductal carcinoma in situ (DCIS) of the breast in relation to mammography: a dilemma. J Natl Cancer Inst Monogr 22: 151 - 156

Ernster VL, Barclay J, Kerlikowske K, Grady D, Henderson C (1996) Incidence of and treatment for ductal carcinoma in situ of the breast [see comments]. JAMA 275: 913-918

Fox SB, Smith K, Hollyer J, Greenall M, Hastrich D, Harris AL (1994) The epidermal growth factor receptor as a prognostic marker: results of 370 patients and review of 3009 patients. Breast Cancer Res Treat 29: $41-49$

Gerweck LE (1998) Tumour pH: implications for treatment and novel drug design. Semin Radiat Oncol 8: 176-182

Ivanov S, Liao SY, Ivanova A, Danilkovitch-Miagkova A, Tarasova N, Weirich G, Merrill MJ, Proescholdt MA, Oldfield EH, Lee J, Zavada J, Waheed A, Sly W, Lerman MI, Stanbridge EJ (2001) Expression of hypoxia-inducible cell-surface transmembrane carbonic anhydrases in human cancer. Am J Pathol 158: 905-919

Ivanov SV, Kuzmin I, Wei MH, Pack S, Geil L, Johnson BE, Stanbridge EJ, Lerman MI (1998) Down-regulation of transmembrane carbonic anhydrases in renal cell carcinoma cell lines by wild-type von HippelLindau transgenes. Proc Natl Acad Sci USA 95: 12 596-12 601

Kivela A, Parkkila S, Saarnio J, Karttunen TJ, Kivela J, Parkkila AK, Waheed A, Sly WS, Grubb JH, Shah G, Tureci O, Rajaniemi H (2000a) Expression of a novel transmembrane carbonic anhydrase isozyme XII in normal human gut and colorectal tumours. Am J Pathol 156: $577-584$

Kivela AJ, Parkkila S, Saarnio J, Karttunen TJ, Kivela J, Parkkila AK, Pastorekova S, Pastorek J, Waheed A, Sly WS, Rajaniemi H (2000b) Expression of transmembrane carbonic anhydrase isoenzymes IX and XII in normal human pancreas and pancreatic tumours. Histochem Cell Biol 114: $197-204$

Leek RD, Landers RJ, Harris AL, Lewis CE (1999) Necrosis correlates with high vascular density and focal macrophage infiltration in invasive carcinoma of the breast. Br J Cancer 79: $991-995$

Liao SY, Stanbridge EJ (1996) Expression of the MN antigen in cervical papanicolaou smears is an early diagnostic biomarker of cervical dysplasia. Cancer Epidemiol Biomarkers Prev 5: 549-557

Maxwell PH, Dachs GU, Gleadle JM, Nicholls LG, Harris AL, Stratford IJ, Hankinson O, Pugh CW, Ratcliffe PJ (1997) Hypoxia-inducible factor-1 modulates gene expression in solid tumours and influences both angiogenesis and tumour growth. Proc Natl Acad Sci USA 94: 8104-8109

Mueller-Klieser W, Freyer JP, Sutherland RM (1983) Evidence for a major role of glucose in controlling development of necrosis in EMT6/Ro multicell tumour spheroids. Adv Exp Med Biol 159: 487-495
Nogradi A (1998) The role of carbonic anhydrases in tumours [comment]. Am J Pathol 153: $1-4$

Opavsky R, Pastorekova S, Zelnik V, Gibadulinova A, Stanbridge EJ, Zavada J, Kettmann R, Pastorek J (1996) Human MN/CA9 gene, a novel member of the carbonic anhydrase family: structure and exon to protein domain relationships. Genomics 33: $480-487$

Parliament MB, Franko AJ, Allalunis-Turner MJ, Mielke BW, Santos CL, Wolokoff BG, Mercer JR (1997) Anomalous patterns of nitroimidazole binding adjacent to necrosis in human glioma xenografts: possible role of decreased oxygen consumption. Br J Cancer 75: $311-318$

Raghunand N, He X, van Sluis R, Mahoney B, Baggett B, Taylor CW, PaineMurrieta G, Roe D, Bhujwalla ZM, Gillies RJ (1999) Enhancement of chemotherapy by manipulation of tumour $\mathrm{pH}$. $\mathrm{Br}$ J Cancer 80: $1005-$ 1011

Ramanujan S, Koenig GC, Padera TP, Stoll BR, Jain RK (2000) Local imbalance of proangiogenic and antiangiogenic factors: a potential mechanism of focal necrosis and dormancy in tumours. Cancer Res 60: $1442-1448$

Saarnio J, Parkkila S, Parkkila AK, Haukipuro K, Pastorekova S, Pastorek J, Kairaluoma MI, Karttunen TJ (1998) Immunohistochemical study of colorectal tumours for expression of a novel transmembrane carbonic anhydrase, MN/CA IX, with potential value as a marker of cell proliferation [see comments]. Am J Pathol 153: 279-285

Shweiki D, Itin A, Soffer D, Keshet E (1992) Vascular endothelial growth factor induced by hypoxia may mediate hypoxia-initiated angiogenesis. Nature 359: $843-845$

Tabar L, Chen HH, Duffy SW, Yen MF, Chiang CF, Dean PB, Smith RA (2000) A novel method for prediction of long-term outcome of women with T1a, T1b, and 10-14 mm invasive breast cancers: a prospective study. Lancet 355: 429-433

Tureci O, Sahin U, Vollmar E, Siemer S, Gottert E, Seitz G, Parkkila AK, Shah GN, Grubb JH, Pfreundschuh M, Sly WS (1998) Human carbonic anhydrase XII: cDNA cloning, expression, and chromosomal localization of a carbonic anhydrase gene that is overexpressed in some renal cell cancers. Proc Natl Acad Sci USA 95: 7608-7613

Vaupel P, Hoeckel M (1999) Predictive power of the tumour oxygenation status. Adv Exp Med Biol 471: 533-539

Vermylen P, Roufosse C, Burny A, Verhest A, Bosschaerts T, Pastorekova S, Ninane V, Sculier JP (1999) Carbonic anhydrase IX antigen differentiates between preneoplastic malignant lesions in non-small cell lung carcinoma. Eur Respir J 14: 806-811

Wykoff CC, Beasley NJP, Watson PH, Turner KJ, Pastorek J, Wilson GD, Turley H, Maxwell PH, Ratcliffe P, Harris AL (2000) Hypoxia inducible expression of tumour associated carbonic anhydrases. Cancer Res 60:7075-7083

Wykoff CC, Beasley N, Watson PH, Campo L, Chia SK, English R, Pastorek J, Sly WS, Ratcliffe P, Harris AL (2001) Expression of the hypoxiainducible and tumour-associated carbonic anhydrases in ductal carcinoma in situ of the breast. Am J Pathol 158: $1011-1019$

Zhong H, De Marzo AM, Laughner E, Lim M, Hilton DA, Zagzag D, Buechler P, Isaacs WB, Semenza GL, Simons JW (1999) Overexpression of hypoxia-inducible factor 1alpha in common human cancers and their metastases. Cancer Res 59: 5830-5835 